\title{
Exposição humana à vibrações de corpo inteiro em um trator agrícola com pneus radiais
}

\author{
Human exposure to whole body vibrations on a farm tractor with radial-ply tires
}

\author{
Rodrigo Lampert Ribas ${ }^{I^{*}}$ José Fernando Schlosser $^{\mathrm{II}}$ Ulisses Giacomini Frantz ${ }^{\mathrm{III}}$ \\ Marcelo Silveira de Farias $^{\mathrm{I}}$ Fabrício Azevedo Rodrigues ${ }^{\mathrm{I}}$
}

RESUMO

O objetivo deste trabalho foi avaliar as vibrações de corpo inteiro incidentes em um operador de trator agrícola utilizando pneus radiais, com três diferentes pressões de insuflagem em operação de semeadura. O trabalho foi divido em três tratamentos, com quatro repetições cada. Utilizou-se um trator Massey Ferguson 5450 Dyna 4, 4x2 TDA, 75kW, acoplado a uma semeadora da mesma marca, modelo MF 509. Os pneus radiais utilizados foram Michelin VF 480/60 R28 para o eixo dianteiro e VF 600/60 R38 para o traseiro, nas pressões de 41, 69 e $96 \mathrm{kPa}$. O equipamento para aquisição dos dados foi o Type 4447 da Brüel \& Kjær, mesma marca do software para análise, o 4447 Vibration Explorer. Todos os tratamentos apresentaram resultados que ficaram na faixa de extremamente desconfortável, segundo os limites considerados pela Norma ISO 2631. A análise estatística não identificou diferença significativa quando a pressão de insuflagem do pneu foi alterada. O eixo X, horizontal frontal, apresentou os maiores valores de vibração incidentes no operador. Concluiu-se que, para uma jornada de 8 horas de trabalho, o operador está exposto acima do nível limite de conforto estabelecido pela norma utilizada.

Palavras-chave: ergonomia, pressão de insuflagem, pneus agrícolas.

\section{ABSTRACT}

The aim of this study was to evaluate the whole body vibrations incidents in a tractor operator using radial ply tires, with three different inflation pressures in operation of seeding. The study was divided into three treatments with four replicates each. Was used a tractor Massey Ferguson 5450, TDA 4x2, 75kW, connected to a planter of the same brand, model 509. Radial ply tires were used VF Michelin 480/60 R28 for the front axle and VF 600/60 R38 for the rear, at pressures of 41, 69 and $96 \mathrm{kPa}$. The equipment for data acquisition was the 4447 Type of Brüel \& Kjær, same brand of software for analysis, the 4447 Vibration Explorer. All treatments showed results that were in the range extremely uncomfortable, according to the limits considered by International standard 2631. Statistical analysis identified no significant difference when the inflation pressure of the tire was changed. The $X$, horizontal forward axis was the one with the highest incidents of vibration to the operator. It was concluded that for an 8-hour workday work, the operator is exposed above the level of comfort limit established by the 2631 International Standard.

Key words: ergonomics, inflation pressure, agricultural tires.

\section{INTRODUÇÃO}

A mecanização agrícola é essencial quando pensamos no modelo de produção da agricultura moderna. Nesse contexto, uma das máquinas que pode ser considerada como a base da agricultura atual é o trator agrícola. Este destaca-se no processo de desenvolvimento da agricultura brasileira, devido à sua versatilidade na execução de inúmeras tarefas no meio rural, servindo como fonte de potência e tração (ROZIN, 2004).

O operador de tratores agrícolas é submetido a uma a jornada de trabalho em torno de 8h diárias, com isso, o conforto e a segurança são de fundamental importância. Nesse sentido, a otimização dos fatores ergonômicos é capaz de aumentar a eficiência com que o sistema homem-máquina executa as suas funções (DEBIASI et al., 2004).

Além dos efeitos nocivos causados pela falta de qualidade ergonômica dos tratores agrícolas,

IPrograma de Pós-graduação em Engenharia Agrícola (PPGEA), Universidade Federal de Santa Maria (UFSM), 97105-900, Santa Maria, RS, Brasil. E-mail: rodrigoribasagronomia@yahoo.com.br*Autor para correspondência.

IIDepartamento de Engenharia Rural (DER), Centro de Ciências Rurais (CCR), UFSM, Santa Maria, RS, Brasil.

IIIUniversidade Federal do Pampa (UNIPAMPA), Dom Pedrito, RS, Brasil. 
a elevada jornada de trabalho que o operador permanece nas atividades agrícolas, principalmente durante a época de safra, torna-se bastante árdua, uma vez que está sujeito a poeiras, vibrações, intempéries e ruídos (IIDA, 2005).

A ergonomia é a ciência que estuda o melhor meio de adaptar o trabalho, seus instrumentos, equipamentos, máquinas e dispositivos para o trabalhador, pela análise do ciclo de trabalho do operador, visando, assim, melhorar o ambiente, para que seja mais seguro e confortável (SILVA et al., 2011).

A análise objetiva para verificar o conforto do operador é feita pela determinação da amplitude, direção, frequência e duração das vibrações. Mesmo que a maior parte da vibração seja transmitida ao operador através do assento, outros mecanismos a influenciam, como é o caso dos pneus, coxins, plataforma, tipo de equipamento que está sendo tracionado, etc.

Os pneus dos tratores agrícolas são responsáveis pelo amortecimento das vibrações, uma vez que os sistemas de amortecimentos mais sofisticados são muito limitados, em nível comercial (HILBERT et al., 2004). Assim, a modificação nos parâmetros relativos aos pneus pode ser uma alternativa para diminuir as vibrações que chegam ao operador e, além disso, conforme WULFSOHN et al. (1988), as características de construção do pneu radial permitem maiores vantagens de tração, sendo resultado da maior deflexão e melhor distribuição de pressão.

A vibração é um movimento oscilatório de um corpo, o qual é devido a forças desequilibradas de componentes rotativos e movimentos alternados de uma máquina ou equipamento (SALIBA et al., 2002). Para a determinação da vibração no corpo humano, existem quatro fatores físicos importantes que são: a intensidade, a frequência, a direção e a duração (tempo de exposição) da vibração (ANFLOR, 2003).

As vibrações de corpo inteiro (VCI) são de baixa frequência e alta amplitude, e situam-se na faixa de 1 a $80 \mathrm{~Hz}$, mais especificamente 1 a $20 \mathrm{~Hz}$. A VCI acontece quando há uma vibração dos pés (posição em pé) ou do assento (posição sentada). Estas vibrações ocorrem mais nas atividades de transporte e vibrações transmitidas por máquinas industriais, e são normatizadas pela Norma ISO 2631 (1997).

Nesse sentido, é necessário o estudo das vibrações que chegam ao posto do operador e sua quantificação, de forma a contribuir para melhorar os fatores ergonômicos. Sendo assim, objetivo deste trabalho foi avaliar as vibrações de corpo inteiro, incidentes no operador de um trator agrícola equipado com pneus radiais, utilizando três diferentes pressões de trabalho em operação de semeadura.

\section{MATERIAL E MÉTODOS}

O trabalho foi realizado em uma área experimental localizada no Campus da Universidade Federal de Santa Maria, RS, Brasil. O tipo de solo predominante na área é o Argissolo Vermelho distrófico com relevo plano suavemente ondulado, sendo que sua umidade, de $11 \%$, foi determinada através da coleta de seis amostras de solo $(0-20 \mathrm{~cm})$. Para determinação do índice de matéria seca da área, que se encontrava com vegetação espontânea, anteriormente ocupada com pecuária de corte, coletou-se o equivalente a uma área de $1 \mathrm{~m}^{2}$ chegando ao valor de $10.500 \mathrm{~kg} \mathrm{ha}^{-1}$.

O delineamento experimental utilizado foi inteiramente casualizado com três tratamentos, nos quais se variou a pressão de insuflagem do pneu e quatro repetições, totalizando 12 unidades experimentais (UEs). As pressões de insuflagem utilizadas foram de 41 (baixa), 69 (recomendada) e 96 (alta) kPa. Utilizouse um trator da marca Massey Ferguson, modelo 5450 Dyna-4, com $75 \mathrm{~kW}$ de potência bruta no motor, 4x2 TDA, configurado com pneus radiais Michelin dianteiros VF 480/60 R28 e traseiros VF 600/60 R38. Este trator tracionava uma semeadora marca Massey Ferguson, modelo MF 509M45, com nove linhas de semeadura, sendo utilizadas no trabalho apenas sete, e sulcador do tipo disco duplo para sementes e haste sulcadora para deposição de fertilizantes. Utilizou-se esse número de linhas em função do tipo de solo existente na área de cultivo e condições de uso. A marcha da transmissão do trator foi a $2^{\text {a }}$ do grupo C, a uma rotação de 2000rpm no motor, o que proporcionou uma velocidade média de deslocamento de $6,4 \mathrm{~km} \mathrm{~h}^{-1}$. Durante o decorrer da aplicação dos tratamentos, foi medido o patinamento médio das rodas do trator, sendo este de 13,46\% para a Pressão de insuflagem Baixa, 11,00\% para a Pressão Recomendada e 21,83\% para a Pressão Alta, semelhante ao encontrado por SERRANO (2008).

Para a aquisição dos dados, foi utilizado o Analisador de Vibrações no Corpo Humano, modelo 4447, da Brüel \& Kjær, que possibilita efetuar medições das vibrações transmitidas ao Corpo Humano Inteiro, situadas na faixa de 1 a $80 \mathrm{~Hz}$ (WBV - Whole Body Vibration). Este equipamento satisfaz exigência da norma ISO 8041 (2005), que determina os padrões a serem seguidos pelas instrumentações de medição.

Conforme a norma ISO 2631 (1997), é necessário que a coleta dos dados seja realizada na interface entre a região do corpo e a superfície vibrante, neste caso, o acelerômetro deve estar inserido na interface do assento-operador. Para isso, utilizou-se um suporte circular semi-rígido (Seat $\mathrm{Pad}$ ), para o posicionamento do acelerômetro e para 
que a pressão seja constante na superfície de contato assento-operador.

O assento do operador foi configurado na posição média, relativas à pressão de mola e altura do assento. Ele possuía o apoio para os braços e um revestimento estofado, fatores estes que contribuem para minimizar as vibrações incidentes no operador.

Após a coleta dos dados, eles foram exportados para o programa 4447 Vibration Explorer 2.0.1, também da Brüel \& Kjær, onde foram tabulados. O programa utilizado para realização da análise de variância e teste de Tukey a 1\% de probabilidade de erro foi o software Sisvar (FERREIRA, 2008).

\section{RESULTADOS E DISCUSSÃO}

Na tabela 1, seguem os principais valores obtidos através da ANOVA realizada. Como é possível observar, não se obteve diferença significativa nos valores de aceleração quando modificada a pressão de insuflagem dos pneus.

Os valores de aceleração eficaz total (RMS VTV), para os tratamentos aplicados, variaram entre $10,73 \mathrm{~m} \mathrm{~s}^{-2}$ e $11,80 \mathrm{~m} \mathrm{~s}^{-2}$, sendo que, na figura 1 , observase que, quanto maior a pressão de insuflagem do pneu, maiores são os índices de vibrações incidentes nos operadores. Porém, quando se realizou a análise estatística, não se identifica diferença significativa entre os tratamentos.

Para os valores médios das acelerações eficazes (RMS), todos os tratamentos apresentaram resultados que ficaram na faixa de Extremamente Desconfortável, segundo os limites considerados pela norma ISO 2631 (1997). Um dos fatores que pode ter contribuído para estes altos valores é a utilização da semeadora com sulcador de adubo do tipo haste fixa.

Avaliando a operação de gradagem do solo, FERNANDES (2003) encontrou valores de aceleração vertical no assento do operador em frequências de ressonância que podem afetar a coluna vertebral do operador. Conforme PINHO (2012), há necessidade de reduzir a magnitude de vibração tridimensional (nos três eixos) no assento de tratores, quando se executam operações de escarificação. Ainda, o autor verificou que os limites estabelecidos pela Norma ISO 2631 (1997) foram excedidos, devido à sensibilidade que o corpo humano apresenta, quando submetido aos efeitos vibratórios. Já DEBOLI et al. (2008) estudou três diferentes pressões e os resultados mostraram que, quanto menor a pressão de trabalho, menores são as acelerações.

Resultados obtidos por CUNHA et al. (2009) evidenciaram que as acelerações no sentido do deslocamento do trator (eixo X) mostraramse superiores às acelerações verticais (eixo Z) e transversais (eixo Y) ao sentido de deslocamento do trator, ultrapassando os valores toleráveis, segundo a norma ISO 2631 (1997).

Para SOLECKI (2007), a análise dos valores máximos e mínimos de pico, de aceleração de vibração, confirma que, no ambiente de trabalho agrícola, ocorre uma variação considerável nos valores registrados de vibração, e que também é evidenciado pelos coeficientes de pico de elevado, por vezes superior a $10 \mathrm{~m} \mathrm{~s}^{-2}$.

Os valores de Ponto de Máximo (Figura 2) também atingiram um alto nível nas três direções (vertical, longitudinal e transversal), ficando com os valores entre $14,85 \mathrm{~m} \mathrm{~s}^{-2}$ e $21,64 \mathrm{~m} \mathrm{~s}^{-2}$. Apesar dos

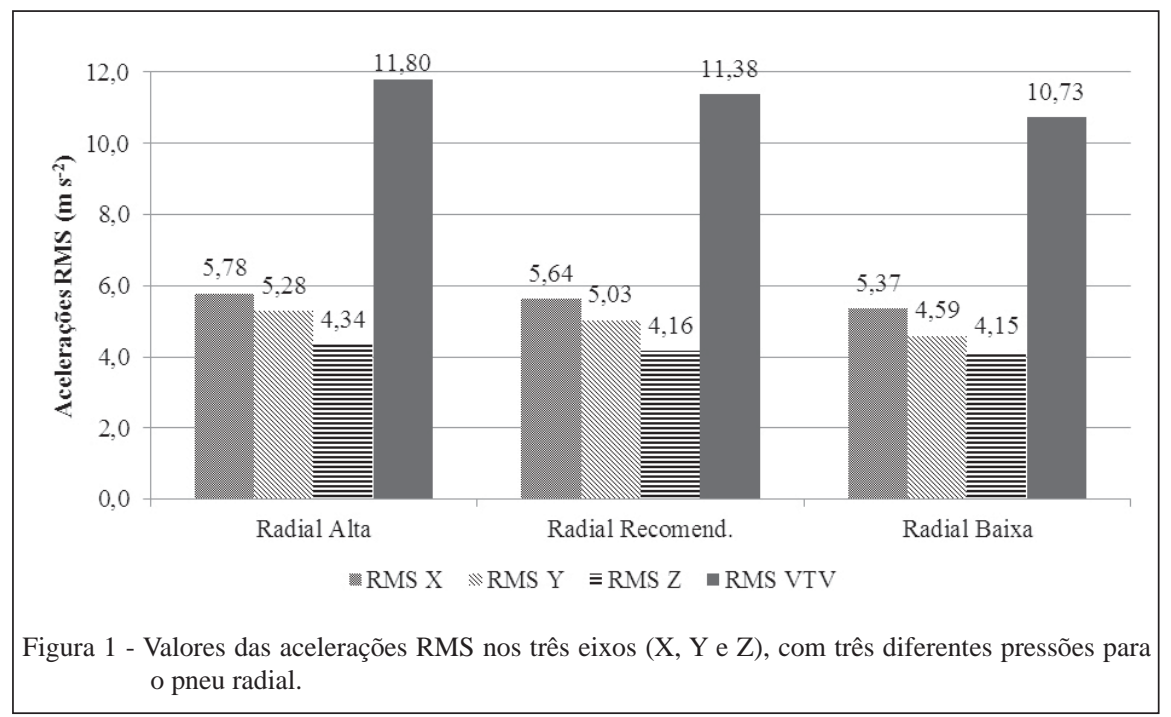

Ciência Rural, v.44, n.9, set, 2014. 
Tabela 1 - ANOVA dos valores de RMS, Ponto de Máximo, VDV e A(8) para o pneu radial.

\begin{tabular}{|c|c|c|c|c|c|}
\hline Variável & Tratamentos & Média & CV (\%) & Fc & $\mathrm{Pr}>\mathrm{Fc}$ \\
\hline \multirow{3}{*}{ RMS X } & $\mathrm{T} 1$ & 5,7800 a & \multirow{3}{*}{5,95} & \multirow{3}{*}{1,586} & \multirow{3}{*}{0,2570} \\
\hline & $\mathrm{T} 2$ & $5,6400 \mathrm{a}$ & & & \\
\hline & T3 & 5,3675 a & & & \\
\hline \multirow{3}{*}{ RMS Y } & $\mathrm{T} 1$ & 5,2850 a & \multirow{3}{*}{6,61} & \multirow{3}{*}{4,621} & \multirow{3}{*}{0,0416} \\
\hline & $\mathrm{T} 2$ & 5,0325 a & & & \\
\hline & T3 & 4,5875 a & & & \\
\hline \multirow{3}{*}{ RMS Z } & $\mathrm{T} 1$ & 4,3375 a & \multirow{3}{*}{8,52} & \multirow{3}{*}{0,361} & \multirow{3}{*}{0,7064} \\
\hline & $\mathrm{T} 2$ & 4,1600 a & & & \\
\hline & $\mathrm{T} 3$ & 4,1425 a & & & \\
\hline \multirow{3}{*}{ RMS VTV } & $\mathrm{T} 1$ & 11,7950 a & \multirow{3}{*}{4,39} & \multirow{3}{*}{4,690} & \multirow{3}{*}{0,0402} \\
\hline & $\mathrm{T} 2$ & 11,3825 a & & & \\
\hline & T3 & 10,7300 a & & & \\
\hline \multirow{3}{*}{ Ponto de Máx X } & $\mathrm{T} 1$ & 21,6375 a & \multirow{3}{*}{18,37} & \multirow{3}{*}{0,107} & \multirow{3}{*}{0,8993} \\
\hline & $\mathrm{T} 2$ & 20,5475 a & & & \\
\hline & T3 & 20,5475 a & & & \\
\hline \multirow{3}{*}{ Ponto de Máx Y } & $\mathrm{T} 1$ & $19,0200 \mathrm{a}$ & \multirow{3}{*}{14,20} & \multirow{3}{*}{0,865} & \multirow{3}{*}{0,4534} \\
\hline & $\mathrm{T} 2$ & 18,5800 a & & & \\
\hline & T3 & 16,7625 a & & & \\
\hline \multirow{3}{*}{ Ponto de Máx Z } & $\mathrm{T} 1$ & $16,9025 \mathrm{a}$ & \multirow{3}{*}{11,54} & \multirow{3}{*}{1,458} & \multirow{3}{*}{0,2604} \\
\hline & $\mathrm{T} 2$ & 16,8450 a & & & \\
\hline & T3 & 14,8475 a & & & \\
\hline & $\mathrm{T} 1$ & 22,0725 a & & & \\
\hline VDV $\mathrm{X}$ & $\mathrm{T} 2$ & 21,3825 a & 6,35 & 0,696 & 0,5237 \\
\hline & T3 & 20,9450 a & & & \\
\hline & $\mathrm{T} 1$ & $20,2175 \mathrm{a}$ & & & \\
\hline VDV Y & $\mathrm{T} 2$ & 19,1200 a & 7,30 & 3,252 & 0,0865 \\
\hline & $\mathrm{T} 3$ & 17,7200 a & & & \\
\hline & $\mathrm{T} 1$ & $16,6900 \mathrm{a}$ & & & \\
\hline VDV Z & $\mathrm{T} 2$ & 15,9375 a & 9,09 & 0,488 & 0,6292 \\
\hline & $\mathrm{T} 3$ & 15,7125 a & & & \\
\hline & $\mathrm{T} 1$ & $45,1475 \mathrm{a}$ & & & \\
\hline VDV VTV & $\mathrm{T} 2$ & $43,1650 \mathrm{a}$ & 4,50 & 3,239 & 0,0872 \\
\hline & $\mathrm{T} 3$ & 41,6500 a & & & \\
\hline & $\mathrm{T} 1$ & 8,0900 a & & & \\
\hline$A(8)$ & $\mathrm{T} 2$ & 7,9525 a & 5,67 & 1,836 & 0,2144 \\
\hline & T3 & 7,5125 a & & & \\
\hline
\end{tabular}

Médias seguidas da mesma letra minúscula na vertical não diferem a 1\% de significância, pelo teste de Tukey.

RMS é o valor eficaz da aceleração nos eixos X, Y, Z e Total (VTV); o Ponto de Máx é o maior valor obtido durante a medição nos eixos X, Y e Z; VDV é o valor da dose de vibração nos eixos X, Y, Z e total (VTV) e A(8) é o valor eficaz total para uma jornada de 8 horas/dia. 


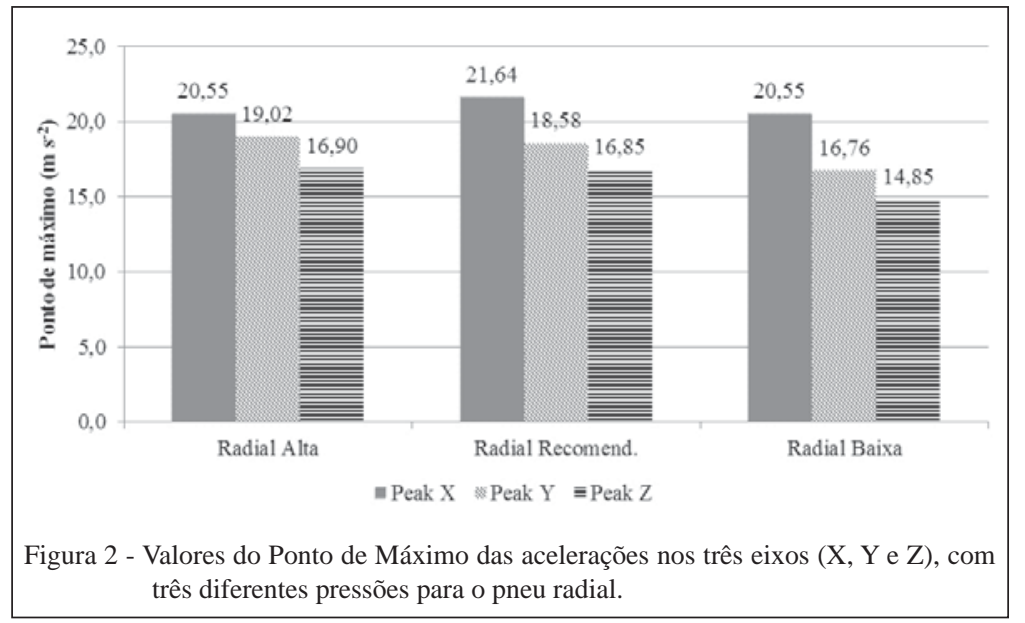

dados mostrarem uma tendência de maiores valores para a pressão mais alta, estatisticamente não foi encontrada diferença significativa.

Com relação aos resultados de valor de dose de vibração (VDV) (Figura 3), os valores encontrados situaram-se entre $41,65 \mathrm{~m} \mathrm{~s}^{-1,75}$ e $45,15 \mathrm{~m}$ $\mathrm{s}^{-1,75}$, o que causa um severo desconforto ao operador. No entanto, estes valores não apresentaram diferença significativa quando analisados estatisticamente.

Corroborando isso, PINHO (2012) comenta que valores de VDV acima de $15 \mathrm{~m} \mathrm{~s}^{-1,75}$ proporcionam severo desconforto ao operador, e que os valores encontrados em seu trabalho variaram de 60 a $202 \mathrm{~m} \mathrm{~s}^{-1,75}$ e, de acordo com os dados analisados, verifica-se que todos os valores ficaram acima do limite estabelecido pelas normas BS 6841 (1987) e BS 7085 (1989). No entanto, ao avaliar a exposição de motoristas de ônibus a vibrações, BALBINOT \& TAMAGNA (2002) verificaram um VDV de 9,7 a $11,5 \mathrm{~m} \mathrm{~s}^{1,75}$. Isso signigica que o operador de tratores agrícolas tem uma maior exposição às vibrações do que os motoristas de ônibus.
Em estudo realizado por FUTATSUKA et al. (1998), estes encontraram os valores de vibração a que os operadores não devem ficar mais de oito horas expostos. Os autores ainda comentam que é necessária a criação de novos mecanismos de suspensão ou redução dos níveis de vibração para máquinas agrícolas.

Todos os tratamentos avaliados neste experimento apresentaram valores de acelerações eficazes superiores aos limites estabelecidos pela norma ISO 2631 (1997), ficando situados na zona considerada Extremamente Desconfortável. Os valores encontrados são tão elevados que estes níveis de vibrações permitiriam que operador pudesse trabalhar na operação de semeadura, no máximo, 20 minutos (Figura 4).

Em um experimento com tratores agrícolas, PINHO (2012) avaliou 36 tratamentos e constatou que as magnitudes das acelerações eficazes, obtidas na interface assento-operador, ficaram acima do limite de conforto na frequência de $1 \mathrm{a} 80 \mathrm{~Hz}$, estabelecidas pela norma ISO 2631 para exposição de oito horas diárias $\left(2,0 \mathrm{~m} \mathrm{~s}^{-2}\right)$.

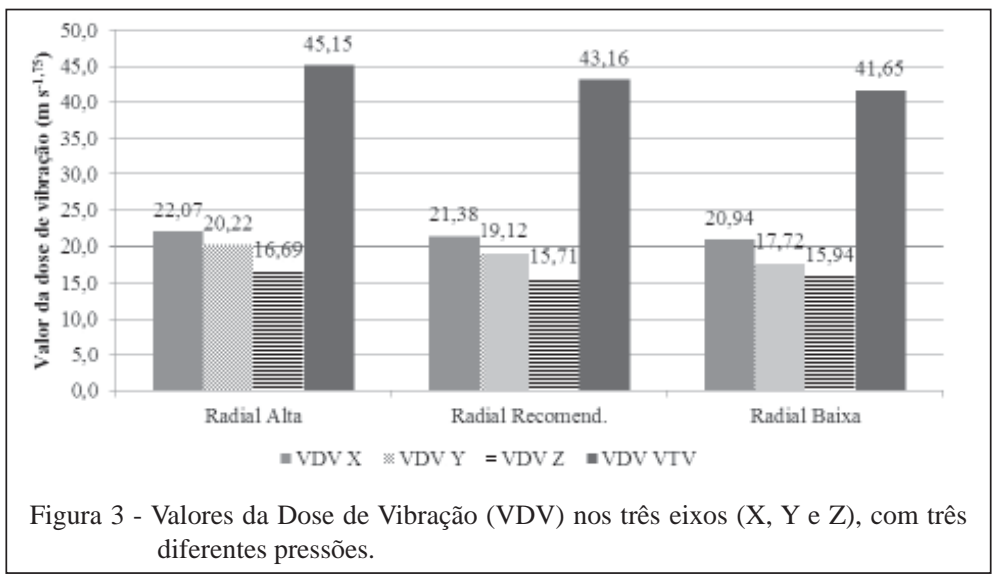

Ciência Rural, v.44, n.9, set, 2014. 


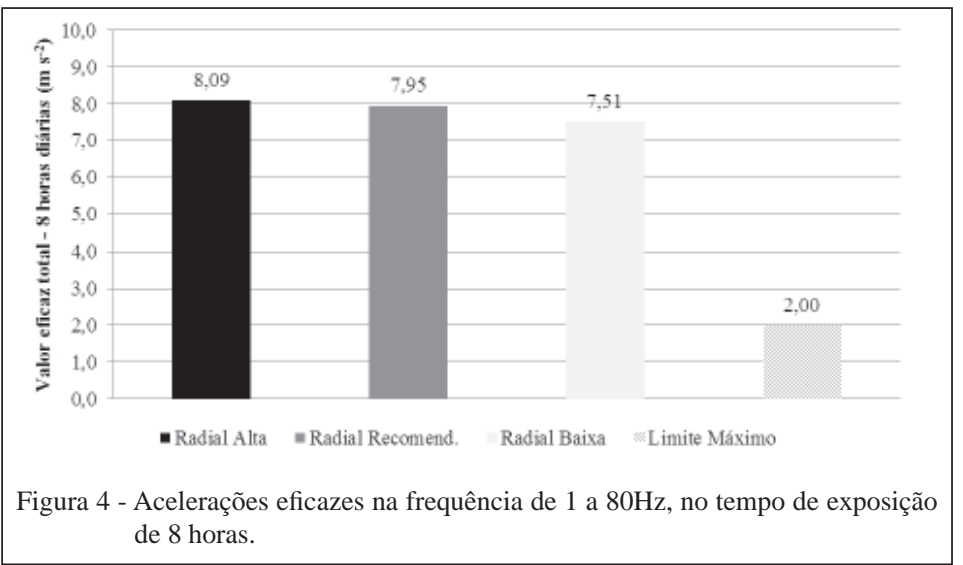

\section{CONCLUSÃO}

Os níveis de vibração referenciados pela Norma ISO 2631 (1997) como limites de conforto do operador foram extrapolados, situando-se na faixa de extremamente desconfortável para os valores eficazes de aceleração (RMS), pelo método RMS;

A direção longitudinal (eixo $\mathrm{X}$ ) foi a que proporcionou ao operador, tanto os maiores valores de acelerações RMS, como de Pico máximo, porém não significativas.

\section{AGRADECIMENTOS}

Os autores agradecem ao apoio da Coordenação de Aperfeiçoamento de Pessoal de Nível Superior (CAPES) e Conselho Nacional de Desenvolvimento Científico e Tecnológico (CNPq) por bolsas de pós-graduação e pela bolsa de produtividade em pesquisa $(\mathrm{CNPq})$ do segundo autor. Também à AGCO do Brasil pela disponibilização do trator e máquina agrícola utilizados neste trabalho.

\section{REFERÊNCIAS}

ANFLOR, C.T.M. Estudo da transmissibilidade da vibração no corpo humano na posição vertical e desenvolvimento de um modelo biodinâmico de quatro graus de liberdade. 2003. 121f. Dissertação (Mestrado em Engenharia) - Curso de pós-graduação em Engenharia Mecânica, Universidade Federal do Rio Grande do Sul, Porto Alegre, RS.

BALBINOT, A.; TAMAGNA, A. Avaliação de transmissibilidade da vibração em bancos de motoristas de ônibus urbanos: um enfoque no conforto e na saúde. Revista Brasileira de Engenharia Biomédica, v.18, n.1, p.31-38, jan./abr. 2002. Disponível em: <http://www.rbeb. org.br/files/v18n1/v18n1a4.pdf>. Acesso em: 12 abr. 2014.

BS 6841. Measurement and evaluation of human exposure to whole-body mechanical vibration and repeated shock. London: British Standard Guide, 1987. 24p.

BS 7085. Safety aspects of experiments in which people are exposed to mechanical vibration and shock. London: British Standard Guide, 1989. 20p.
CUNHA, J.P.A.R. da et al. Avaliação dos níveis de vibração e ruído emitidos por um trator agrícola em preparo de solo. Pesquisa Agropecuária Tropical, v.39, n.4, p.348-355, out./dez. 2009. Disponivel em: <www.revistas.ufg.br/index.php/pat/article/ download/6927/5904>. Acesso em: 12 abr. 2014.

DEBIASI, $\mathrm{H}$. et al. Acidentes de trabalho envolvendo conjuntos tratorizados em propriedades rurais do Rio Grande do Sul, Brasil. Ciência Rural, v.34, n.3, p.779-784, maio/ jun. 2004. Disponível em <http://dx.doi.org/10.1590/S010384782004000300019>. Acesso em: 17 dez. 2013. doi: 10.1590/ S0103-84782004000300019>.

DEBOLI, R. et al. Whole body vibration (WBV) transmitted to the operator by tractors equipped with radial tires. In: INTERNATIONAL CONFERENCE: INNOVATION TECHNOLOGY TO EMPOWER SAFETY, HEALTH AND WELFARE IN AGRICULTURE AND AGRO-FOOD SYSTEMS, 2008, Ragusa. Anais eletrônicos... Ragusa, 2008. Disponível em: <http://www.ragusashwa.it/CD_2008/lavori/TOPIC7/poster/ DEBOLI-1.pdf $>$. Acesso em: 16 nov. 2012.

FERREIRA, D.F. SISVAR: um programa para análises e ensino de estatística. Revista Symposium, v.6, n.2, p.36-41, 2008. Disponível em: <http://www.fadminas.org.br/symposium>. Acesso em: 04 jan. 2013.

FERNANDES, H.C. et al. Vibração em tratores agrícolas: caracterização das faixas de frequência no assento do operador. Engenharia na Agricultura, Viçosa, v.11, n.1-4, p.23-31, 2003. Disponivel em: <http://www.ufv.br/dea/reveng/arquivos/vol11/ v11n1-4p23-31.pdf> . Acesso em: 12 abr. 2014.

FUTATSUKA, M. et al. Whole-body vibration and health effects in the agricultural machinery drivers. Industrial Health, v.36, n.2, p.127-132, abr. 1998. Disponível em: <http://www.ncbi.nlm.nih. gov/pubmed/9583309>. Acesso em: $19 \mathrm{dez}$. 2013. doi: 10.2486/ indhealth.36.127.

HILBERT, J. et al. Estudio de impacto utizando diferentes presiones de inflado sobre las vibraciones de cuerpo entero en tractores agrícolas. In: CONGRESSO BRASILEIRO DE ENGENHARIA AGRÍCOLA, 33., 2002, São Pedro, SP. Anais... São Paulo: Sociedade Brasileira de Engenharia Agrícola, 2004. CD Rom.

IIDA, I. Ergonomia: projeto e produção. 2.ed. São Paulo: Edgard Blucher, 2005. v.1, 614p.

Ciência Rural, v.44, n.9, set, 2014. 
INTERNATIONAL ORGANIZATION FOR STANDARDIZATION (ISO). ISO 2631: Mechanical vibration and shock - evaluation of human exposure to whole-body vibration - Part 1: General guidelines. Geneva, 1997. 31p.

ISO 8041: Human response to vibration - measuring instrumentation. Geneva, 2005. 91p.

PINHO, M. da S. Vibrações em um trator agrícola cabinado: magnitude das acelerações no assento e transmissibilidade através do coxim. 2012. 158f. Tese (Doutorado em Engenharia Agrícola) - Programa de Pós-graduação em Engenharia Agrícola, Universidade Federal de Santa Maria, RS.

ROZIN, D. Conformidade do posto de operação de tratores agrícolas nacionais com Normas de ergonomia e segurança. 2004. 204f. Dissertação (Mestrado em Engenharia Agrícola) Programa de Pós-graduação em Engenharia Agrícola, Universidade Federal de Santa Maria, Santa Maria, RS.

SALIBA, T.M. et al. Higiene do trabalho: programa de prevenção de riscos ambientais. 3.ed. São Paulo: Ltr, 2002. 262p.
SERRANO, J.M.P.R. Pressão de insuflagem dos pneus no desempenho do conjunto trator-grade de discos. Pesquisa Agropecuária Brasileira, v.43, n.2, p.227-233, 2008. Disponível em: <http://dx.doi.org/10.1590/S0100-204X2008000200011>. Acesso em: 17 dez. 2013. doi:10.1590/S0100-204X2008000200011.

SILVA, C.B. et al. Avaliação ergonômica de uma colhedora de cana-deaçúcar. Ciência e Agrotecnologia, v.35, n.1, p.179-185, jan./fev. 2011. Disponível em: <http://dx.doi.org/10.1590/S1413-70542011000100023>. Acesso em: 17 dez. 2013. doi: 10.1590/S1413-70542011000100023.

SOLECKI, L. Preliminary recognition of whole body vibration risk in private farmers' working environment. Annals of Agricultural and Environmental Medicine, v.14, n. 2, p. 299304, dez. 2007. Disponível em: <http://www.ncbi.nlm.nih.gov/ pubmed/18247468>. Acesso em 12 abr. 2014.

WULFSOHN, D. et al. Tractive characteristics of radial ply and bias ply tyres in a californiam soil. Journal of Terramechanics, v.25, n.2, p.111-34, 1988. Disponível em: <http://dx.doi.org/10.1016/00224898(88)90019-5>. Acesso em: 17 dez. 2013. doi: 10.1016/00224898(88)90019-5. 